\title{
Effects of rhythmic sensory stimulation (auditory, visual) on gait in Parkinson's disease patients
}

\author{
Pablo Arias, Javier Cudeiro
}

\begin{abstract}
This study has focused on how sensory stimulation affects gait in Parkinson's disease (PD). The kinematic parameters of gait [cadence, step amplitude, velocity, coefficient of variation of stride time $\left(\mathrm{CV}_{\text {stride-time }}\right)$, and the coefficient of variation of the step amplitude $\left.\left(\mathrm{CV}_{\text {step-amplitude }}\right)\right]$ were analysed in 25 $\mathrm{PD}$ patients and 10 control subjects. Step amplitude, velocity and $\mathrm{CV}_{\text {stride-time }}$ were altered in the patients with PD. However, when kinematic parameters were analysed as a function of disease severity, none of the parameters differed between early PD (I-II Hoehn and Yahr) and the controls. Nevertheless, more severely affected PD patients (III-IV Hoehn and Yahr) walked with a reduced step amplitude, lower velocity, higher $\mathrm{CV}_{\text {stride-time, }}$ and higher $\mathrm{CV}_{\text {step-amplitude }}$ than the controls. The administration of auditory stimulation at a frequency matching the preferred walking cadence led to a decrease in the $\mathrm{CV}_{\text {stride-time }}$ in $\mathrm{PD}_{\text {III-IV }}$ patients, and to an increase in step amplitude in $\mathrm{PD}_{\text {III-IV }}$ and controls. Visual stimulation at the same frequency did not modify any of the altered kinematic parameters in $\mathrm{PD}_{\text {III-IV }}$ patients. When different stimulation frequencies were utilised, auditory stimulation significantly changed some of the altered walking parameters in Parkinson patients. Frequencies matching preferred walking cadence or above this, up to the fastest walking, were those that seem to interact most effectively with the abnormal kinematic parameters in PD $\mathrm{PIIIV}_{\mathrm{IV}}$ patients. Visual stimulation negatively modulated cadence in $\mathrm{PD}_{\text {III-IV }}$ in the frequency range used. Sensory stimulation facilitates gait in PD. Studies using sensory stimulation as a tool to facilitate walking should take into account the grade of disability of the patients.
\end{abstract}

Keywords. Parkinson, Gait, Sensory stimulation, Rhythm

\section{Introduction}

The gait of patients suffering from PD is characterized by the alteration of different spatio-temporal parameters. One of these alterations is a shorter step/stride length compared to control subjects, which is responsible for the decreased velocity manifested by these patients (Blin et al. 1990; Morris et al. 1994a, 1996, 2005; Thaut et al. 1996; Azulay et al. 1999; Ebersbach et al. 1999; Lewis et al. 2000; Frenkel-Toledo et al. 2005; Fernández del Olmo and Cudeiro 2005; Rochester et al. 2005; Willems et al. 2006). Coefficient of variation (CV) of stride length is also thought to increase (Blin et al. 1990; Lewis et al. 2000). While the shorter stride is considered the main feature of the Parkinsonian gait (Morris et al. $1994 a, b, 1998)$, alterations in cadence seem to be more controversial. While several studies have shown that cadence remains unaltered in PD (Morris et al. 1994a, 1998, 2005; Hausdorff et al. 1998; Lewis et al. 2000; Fernández del Olmo and Cudeiro 2005; Rochester et al. 2005; Frenkel-Toledo et al. 2005) others have detected a reduction in cadence (Blin et al. 1990; Thaut et al. 1996; Azulay et al. 1999; Ebersbach et al. 1999; Willems et al. 2006). A high coefficient of variation for the stride cycle time ( $\left.\mathrm{CV}_{\text {stride-time }}\right)$ is also an important feature of PD gait (Hausdorff et al. 1998; Fernández del Olmo and Cudeiro 2005), as it is considered an indicator of the risk of suffering from falls (Schaafsma et al. 2003).

The PD is a progressive disease in which the alterations in gait vary according to the severity of the disease (Goetz et al. 2004). Thus, when characterizing PD gait it is necessary to take into account the phase in which the patients are at the time of analysis, which has not always been the case.

Previous studies have shown that the utilization of rhythmic sensory cues facilitates walking in PD and that PD patients walk better in the presence of rhythmic auditory cues (Enzensberger and Fischer 1996; McIntosh et al. 1997; Howe et al. 2003). While some studies have focused on the effect of auditory stimulation at a frequency matching preferred walking cadence (PW-cadence) (Freedland et al. 2002; Rochester et al. 2005; Willems et al. 2006), others have used different stimulation frequencies 
(Enzensberger and Fischer 1996; McIntosh et al. 1997; Ebersbach et al. 1999; Freedland et al. 2002; Howe et al. 2003; Willems et al. 2006), with baseline condition (PW or fastest-walking-FW) differing between studies. The observed improvement in performance is manifested by the modification of parameters such as amplitude or velocity, and is seen with frequencies higher than PW-cadence. Despite these improvements, attempts to use stimulation protocols which search across a range of frequencies in order to identify the best stimulation parameters to be used, are scarce, [however, see Willems et al. (2006) and Howe et al. (2003)].

Visual stimulation has also been used in order to improve the walking pattern of PD patients. Traditionally, visual stimuli were provided by means of a series of stripes laid out on the floor for the patients to walk over. This kind of stimulation has been shown to improve stride amplitude (Morris et al. 1994a, 1996, 2005; Azulay et al. 1999; Lewis et al. 2000; Suteerawattananon et al. 2004), velocity (Morris et al. 1996, 2005; Azulay et al. 1999; Lewis et al. 2000) and the CV of stride amplitude (Lewis et al. 2000). However, this type of stimulation is not practical to apply outside of a controlled environment, despite the efforts made by Lewis et al. (2000). Recently, rhythmic visual stimulation has been proposed provided by light emitting diode’s (LED) integrated in a pair of goggles (Rochester et al. 2005).

In addition, some studies have focused on the potential of training paradigms, particularly using auditory stimuli, in which patients improve gait, and in some cases, make long-lasting improvement (Miller et al. 1996; Thaut et al. 1996; Marchese et al. 2000; Fernández del Olmo and Cudeiro 2005; Fernández del Olmo et al. 2006; Nieuwboer et al. 2007).

However, the studies using auditory and visual stimulation carried out to date have not taken into account the grade of the disability of the patients. The work reported here forms part of a series of studies investigating both auditory and visual sensory stimulation in PD patients. The aim of the work was threefold: (1) to characterize Preferred Walking gait in PD patients and to determine the differences according to the grade of development of the disease; (2) to identify the effect of external sensory cues (auditory, visual, and auditory-visual) on the performance of those patients suffering from significant alterations in their walking pattern; (3) to test the effect of applying sensory stimuli at different frequencies in order to determine which frequency produces the best results, with a view to optimizing stimulation strategies for future rehabilitation paradigms. For aim (2), stimulation frequency was set at a rate that matched the cadence of each subject during their PW. In this protocol any change in the walking pattern would therefore be attributable to the presence of the stimulus, not to its frequency, since it matched the PW cadence. For aim (3), two groups of auditory and visual stimulation frequencies were established, optimizing the range to each individual's cadence in the walking test at their fastest speed in the absence of stimulation.

\section{Methods}

Subjects

Patients

The patients were recruited from the Parkinson's Disease Association of Galicia (Table 1) and evaluated from October to December 2005. The study was carried out on 25 idiopathic PD patients (Table 2). In order to take into consideration disease severity when analyzing the data, the whole group was divided into two groups, mild and severe patients. The mild PD group ( $\mathrm{n}=16)$ comprised 16 patients in phases I or II according to the Hoehn and Yahr (1967) evaluation scale ( $\left.\mathrm{PD}_{\mathrm{I}-\mathrm{II}}\right)$. The group of severe patients $(n=9)$ included patients considered as phase III or IV patients $\left(\mathrm{PD}_{\text {III-IV }}\right)$ and these $\mathrm{PD}_{\text {III-IV }}$ patients, were those who participated in the experiments in which the effects of sensory stimulation were assessed.

Prior to the experiment, all patients were examined by a neurologist (who was unaware of the nature of the experiment) using a battery of tests including: the short test of mental status (STMS, Kokmen et al. 1987); UPDRS (Fahn and Elton 1987); Hoehn and Yahr (1967); and Schwab and England (1969) scales. Patients were excluded from the study if they displayed any of the following features:

Neurological disease other than PD; musculoskeletal, cardiovascular, auditory or visual impairment; inability to walk for $30 \mathrm{~m}$; STMS score $<30$.

Evaluation was carried out while patients were on-dose and the session lasted no more than $1 \mathrm{~h}$ to avoid the effects of the medication wearing-off. Tests started about $45 \mathrm{~min}$ after morning dose intake. 
Table 1. Trial flow-chart

Assessed for eligibility

Controls $(n=10)$-PD $(n=37)$

Allocation

(Controls, $n=10$ ) Allocation to intervention (PD, $n=25$ )

(Controls, $n=10$ ) Received allocated intervention (PD, $n=25)$

Excluded: 12 PD

(Controls, $n=0)$ Did not receive allocated intervention $(\mathrm{PD}, \mathrm{n}=0$ )

Not meeting inclusion criteria:

4: <30 STMS

1: Hip Prosthesis

3: Arthritis.

3: Visual impairment (1 Glaucoma, 2 Cataract)

Refused to participate: 0

Other reason: 1 (feeling sick the day of the experiment)

Analysis

(Controls, $n=10$ ) Analysed (PD, $n=25)$

(Controls, $n=0)$ Excluded from analysis (PD, $n=0)$

Table 2. Patient's characteristics

\begin{tabular}{|c|c|c|c|c|c|c|c|}
\hline M.A.L & $\mathrm{M}$ & 51 & 0.89 & 2 & 1 & 90 & 12 \\
\hline C.P.M & $\mathrm{F}$ & 70 & 0.85 & 2 & 2 & 80 & 32 \\
\hline C.S.R & $\mathrm{M}$ & 66 & 0.87 & 5 & 2 & 80 & 38 \\
\hline $\mathrm{JM}^{\mathrm{a}}$.G.R & $\mathrm{M}$ & 69 & 0.80 & 5 & 2 & 80 & 25 \\
\hline R.R.C & $\mathrm{M}$ & 55 & 0.87 & 3 & 2 & 80 & 29 \\
\hline D.R.M & $\mathrm{F}$ & 70 & 0.84 & 13 & 2.5 & 70 & 47 \\
\hline E.G.N & $\mathrm{M}$ & 76 & 0.89 & 6 & 2.5 & 70 & 48 \\
\hline E.S.S & $\mathrm{F}$ & 62 & 0.82 & 7 & 2.5 & 80 & 33 \\
\hline$M^{\mathrm{a}}$ L.M.F & $\mathrm{F}$ & 55 & 0.83 & 12 & 2.5 & 70 & 65 \\
\hline J.E.L & $\mathrm{M}$ & 70 & 0.88 & 10 & 2.5 & 80 & 58 \\
\hline JM ${ }^{a} . P . G$ & $\mathrm{M}$ & 66 & 0.89 & 9 & 3 & 60 & 68 \\
\hline C.V.L & $\mathrm{M}$ & 71 & 0.84 & 8 & 3 & 50 & 78 \\
\hline J.C.I & $\mathrm{F}$ & 75 & 0.82 & 20 & 3 & 40 & 82 \\
\hline M.T.C & $\mathrm{M}$ & 69 & 0.98 & 7 & 3 & 40 & 75 \\
\hline$M^{\mathrm{a}}$.G.A & $\mathrm{F}$ & 73 & 0.81 & 6 & 3 & 50 & 64 \\
\hline M'M.M.S & $\mathrm{F}$ & 71 & 0.80 & 7 & 3 & 60 & 73 \\
\hline G.P.V & $\mathrm{F}$ & 74 & 0.81 & 23 & 3 & 50 & 70 \\
\hline J.C & $\mathrm{M}$ & 68 & 0.86 & 14 & 3 & 40 & 72 \\
\hline \multirow[t]{2}{*}{ A.M.G } & $\mathrm{M}$ & 75 & 0.84 & 22 & 4 & 20 & 84 \\
\hline & & Mean & Mean & Mean & Mean & Mean & Mean \\
\hline $\mathrm{PD}(n=25)$ & & $65.92( \pm 7.65)$ & $0.86( \pm 0.04)$ & $8.96( \pm 6.22)$ & $2.52( \pm 0.60)$ & $65.20( \pm 18.06)$ & $53.40( \pm 21.31)$ \\
\hline
\end{tabular}

\section{Control subjects}

Ten healthy subjects were recruited as the control group. There were no significant differences in age (65.70 years \pm 7.65$)$ and trochantereal height $(0.86 \mathrm{~m} \pm 0.05)$ between PD patients and controls. In the experiments to assess the effects of sensory stimulation, nine of the healthy subjects were sub-selected as controls whose age and trochanteral height did not differ from those of the PD $\mathrm{PIIIIV}_{\mathrm{IV}}$ group.

Subjects were informed about the nature of the test. The protocol was in compliance with the Helsinki declaration and it was approved by the University of A Coruña Ethics Committee. 
Procedure

\section{Characterization of gait pattern}

In the first instance, this study set out to characterize the gait pattern in a population of PD patients and to compare this with the gait displayed by control subjects.

In order to establish the gait patterns in controls and PD patients, subjects were required to walk along a $30 \mathrm{~m}$ level walkway using their preferred walking pattern. Recordings were acquired from the central $25 \mathrm{~m}$, disregarding the $2.5 \mathrm{~m}$ at the beginning and at the end of the walkway to minimize the effect of acceleration and deceleration. The instruction given to the subjects was "Walk to the end of the corridor as you normally do”.

\section{Stimulation at a frequency matching PW-cadence}

Subsequently, we investigated the use of sensory rhythmic stimulation as a tool to facilitate walking in PD patients. Utilising the results of our first study, this kind of stimulation was only used with those patients who suffered a significant alteration in gait and we used only frequencies of stimulation, which matched the normal cadence of each patient.

The subjects were required to walk along the same walkway used before in presence of stimulation at a frequency matching each subject's PW-cadence. Three kinds of stimuli were presented in counterbalanced way: auditory (PWA); visual (PWV); and auditory-visual (PWAV).

\section{Utilization of different stimulation frequencies}

Finally, we introduced different stimulation frequencies in order to identify the best frequencies to be used. Subjects were required to walk along the same walkway first in the absence and then in the presence of sensory stimulation. Stimulation was provided across a range of frequencies from 70 to $110 \%$ in a counterbalanced way, in $10 \%$ increments-decrements, with $100 \%$ defined as the unaided FW cadence of each patient. Two types of stimulus were used, one visual and one auditory (see below).

The FW-cadence was selected as a reference instead of the PW-cadence as this second option would have required setting frequencies above and below the PW-cadence, augmenting the number of tests required. Since the number of conditions that could be tested was limited by the duration of the medication effects, we would be more likely to miss important frequencies if the PW-cadence were used as a reference. FW-cadence has also been shown to be similar between PD and control subjects (Morris et al. 1994a), making the use of this measurement more appealing.

The instruction given to the subject was "Walk to the end of the corridor matching the rhythm of the stimuli, executing one step per stimulus”.

Patients did not expect to obtain any direct and immediate benefit from this experiment as it was considered a system to evaluate gait.

Subjects rested seated for 2.5 min between conditions.

\section{Apparatus}

The system used to record the data consisted of a series of footswitches worn as insoles in the shoes. The footswitches were connected by means of fine wires to a radio-transmitter attached to the subjects' belt. Prior to testing the subjects were asked to move around the room freely, habituating them to the equipment and the environment, while also checking data transmission. Data (sampled at $1 \mathrm{kHz}$.) were sent via radio to a receiver unit connected to the computer. This configuration allowed the stride cycle time to be registered.

Two photocells, placed $25 \mathrm{~m}$ apart, were connected to the recording system so that the recording commenced and terminated when the subjects crossed these at the beginning and at the end of the walkway. A 2D video-analysis of walking was performed by means of software implemented in MatLab 6.0. A Canon-XL1 Camcorder was used at a frame-rate of 25 frames/s (sufficient to get 2-3 doublesupport phase frames), which was attached to a $25 \mathrm{~m}$-travelling system that moved parallel to the walkway. In this way, a video recording was made along the entire walkway, the analysis of which was aided by placing reflective markers at the tip and the heel of each shoe.

To assess the effects of sensory stimulation, the same apparatus was used with the addition of a portable in-house device to provide stimulation. Auditory stimulation was provided by means of headphones. The sound was a tone with wave-frequency of $4,625 \mathrm{~Hz}$, and intensity was adjusted to be 
clearly perceived by the subjects without being annoying. Visual stimulation was provided by 2 LED's integrated into the subject's glasses, so that stimulation is perceived in the peripheral visual field. The LED's were $3 \mathrm{~mm}$ in diameter with a luminance of $50 \mathrm{mcd} / \mathrm{m}^{2}$. The stimuli were delivered in pulses of $50 \mathrm{~ms}$ and the inter-pulse duration was customized to obtain the desired stimulation frequency. In the case of auditory-visual stimulation both stimuli were set in-phase and at the same frequency.

Kinematic variables

The variables analysed in the study were:

Cadence- obtained from footswitch data and expressed as steps/min;

Step amplitude-calculated from the 2D analysis (when gait was characterised and the effect of stimulation at a frequency matching PW-cadence was assessed); and from the distance walked and the number of steps necessary to cover the distance when assessing the effect of using different stimulation frequencies, expressed in m;

Velocity — calculated as the product of the step amplitude and cadence, and expressed as $\mathrm{m} / \mathrm{min}$;

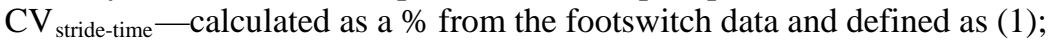

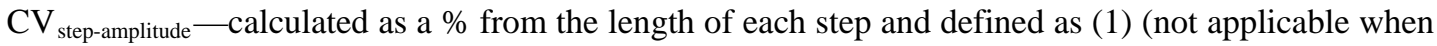
studying the effect of stimuli at frequencies different from PW-cadence).

$\mathrm{CV}(\%)=(\mathrm{SD} / \mathrm{mean}) \times 100(1)$

\section{Statistical analysis}

The student's $t$ test for independent samples was used to analyse the differences between PD and controls for each variable. To assess differences as a function of disease severity one-way ANOVA was performed for each variable, and group was the factor considered ( $\mathrm{PD}_{\mathrm{I}-\mathrm{II}}, \mathrm{PD}_{\mathrm{III}-\mathrm{IV}}$, and controls). The normality of distribution was checked by K-S and the equality of variance by Levene's test. To analyze the effects of stimulation at a frequency matching PW-cadence, repeated measures ANOVA (ANOVARM) was calculated for each kinematic variable. One within-subject factor was established (cue) with four levels: no cue, auditory cue, visual cue, and auditory-visual cue. Additionally, one between-subject factor was set, the group.

To analyze the effect of different stimulation frequencies ANOVA-RM was calculated for each kinematic variable and for each type of stimulation (auditory or visual). One within-subjects factor was set (frequency) with six levels: no cue (i.e. PW), and 70, 80, 90, 100, and $110 \%$ of FW-cadence. One between-subjects factor was set, the group. Pairwise comparisons were only performed with the Controls, since the object of the study was to determine if there were frequencies at which gait induced by stimulation improved kinematic parameters with respect to the PW.

In order to analyse how cadence was modulated as a function of frequency and stimulation varieties an ANOVA-RM was calculated. Two within-subjects factors were used, one (frequency) with five levels: $70,80,90,100$, and $110 \%$; and the other (Cue) with two levels: auditory (cadence in presence of auditory stimulation), and visual (cadence in presence of visual stimulation). The between-subjects factor was the group.

\section{Results}

Gait patterns: patients vs. controls

The kinematic variables measured were compared between the PD patients and the control subjects (Table 3). While there was no difference in the cadence between the groups walking at PW, the PD patients walked with shorter step amplitude $(\mathrm{t}(32.92)=3.003 ; \mathrm{P}=0.005)$ and a reduced velocity $(\mathrm{t}(29.95)=2.160 ; \mathrm{P}=0.039)$ when compared with the controls. Furthermore, there was more variability in the walking pattern of PD patients than in controls, expressed as $\mathrm{CV}_{\text {stride-time }}(\mathrm{t}(26.34)=2.575$; $\mathrm{P}=0.016)$. However, the $\mathrm{CV}_{\text {step-amplitude }}$ at their $\mathrm{PW}$ was no different between patients and controls.

When the PD patients were analysed taking into account the severity of their disease (Table 4), oneway ANOVA showed a main effect of factor group for amplitude $(\mathrm{F}(2,32)=13.154 ; \mathrm{P} \leq 0.001)$, velocity $(\mathrm{F}(2,32)=8.966 ; \quad \mathrm{P}=0.001), \quad \mathrm{CV}_{\text {stride-time }} \quad(\mathrm{F}(2,32)=13.477 ; \quad \mathrm{P} \leq 0.001), \quad$ and $\quad \mathrm{CV}_{\text {step-amplitude }}$ $(\mathrm{F}(2,32)=11.410 ; \mathrm{P} \leq 0.001)$; conversely the effect of factor group for cadence did not show significance. 
Table 3. Kinematic parameters for PD and Controls individuals

\begin{tabular}{llll}
\hline & PD & Controls & Significance \\
\hline & & & \\
Cadence (steps/min) & $114.62( \pm 13.58)$ & $113.73( \pm 14.58)$ & n.s. \\
Step amplitude (m) & $0.54( \pm 0.17)$ & $0.65( \pm 0.06)$ & $P=0.005$ \\
Velocity (m/min) & $61.93( \pm 23.62)$ & $75.28( \pm 12.58)$ & $P=0.039$ \\
CV (\%) of stride time & $4.81( \pm 4.69)$ & $2.33( \pm 0.67)$ & $P=0.016$ \\
CV (\%) step amplitude. & $6.61( \pm 4.88)$ & $3.92( \pm 1.41)$ & n.s. \\
& & & \\
\hline
\end{tabular}

Table 4. Kinematic parameters for $\mathrm{PD}_{\mathrm{I}-\mathrm{II}}, \mathrm{PD}_{\mathrm{III}-\mathrm{IV}}$ and control subjects

\begin{tabular}{|c|c|c|c|c|c|c|}
\hline & \multicolumn{2}{|l|}{$\mathrm{PD}_{\mathrm{I}-\mathrm{II}}$} & \multicolumn{3}{|l|}{$\mathrm{PD}_{\text {III-IV }}$} & \multirow{2}{*}{ Controls } \\
\hline & & $\begin{array}{l}\text { vs. controls } \\
\text { significance }\end{array}$ & $\begin{array}{l}\text { vs. PD } D_{\text {I-II }} \\
\text { significance }\end{array}$ & & $\begin{array}{l}\text { vs. controls } \\
\text { significance }\end{array}$ & \\
\hline Cadence (steps/min) & $115.63( \pm 14.84)$ & n.s. & n.s. & $110.34( \pm 14.31)$ & n.s. & $114.62( \pm 13.58)$ \\
\hline Step amplitude (m) & $0.62( \pm 0.12)$ & n.s. & $P \leq 0.001$ & $0.40( \pm 0.16)$ & $P \leq 0.001$ & $0.66( \pm 0.06)$ \\
\hline Velocity (m/min) & $72.00( \pm 20.19)$ & n.s. & $P \leq 0.001$ & $44.03( \pm 18.61)$ & $P \leq 0.001$ & $75.28( \pm 12.58)$ \\
\hline CV (\%) of stride Time & $2.59( \pm 1.47)$ & n.s. & $P \leq 0.001$ & $8.77( \pm 5.88)$ & $P \leq 0.001$ & $2.33( \pm 0.67)$ \\
\hline CV (\%) step amplitude & $4.41( \pm 1.99)$ & n.s. & $P \leq 0.001$ & $10.52( \pm 6.09)$ & $P \leq 0.001$ & $3.92( \pm 1.41)$ \\
\hline
\end{tabular}

Pairwise comparisons showed there were no significant differences between $\mathrm{PD}_{\mathrm{I}-\mathrm{II}}$ and controls in any of the variables analysed.

Conversely, compared to the controls, there was a reduction in step amplitude and velocity in the more severe $\mathrm{PD}_{\text {III-IV }}$ patients; additionally $\mathrm{PD}_{\text {III-IV }}$ displayed higher $\mathrm{CV}_{\text {stride-time }}$ and a higher $\mathrm{CV}_{\text {step-amplitude }}$ ( $\mathrm{P} \leq 0.001$ for each).

We assessed the differences in the variables measured between the two different groups of PD patients. $\mathrm{PD}_{\text {III-IV }}$ patients took shorter steps and walked slower than $\mathrm{PD}_{\mathrm{I}-\mathrm{II}}$ patients; finally, the $\mathrm{CV}_{\text {stride-time }}$ and the $\mathrm{CV}_{\text {step-amplitude }}$ were higher in severe $\mathrm{PD}_{\text {III-IV }}$ patients than in milder $\mathrm{PD}_{\mathrm{I}-\mathrm{II}}$ patients $(\mathrm{P} \leq 0.001$ for each).

Stimulation conditions

Stimulation at a frequency matching $\mathrm{PW}$-cadence

The effects of sensory stimulation on the kinematics variables of gait were assessed (Table 5).

Table 5. Effect of sensory stimulation on kinematic parameters of gait in $\mathrm{PD}_{\text {III-IV }}$ and control subjects

\begin{tabular}{llllll}
\hline & & PW & PWA & PWV & PWAV \\
\hline \multirow{3}{*}{ PD III-IV } & Cadence (steps/min) & $110.34( \pm 14.31)$ & $108.53^{*}( \pm 11.30)$ & $104.74^{*}( \pm 11.06)$ & $104.79^{* * *}( \pm 12.04)$ \\
& Step amplitude (m) & $0.40( \pm 0.16)$ & $0.45^{* *}( \pm 0.15)$ & $0.41( \pm 0.16)$ & $0.44^{*}( \pm 0.17)$ \\
& Velocity (m/min) & $44.03( \pm 18.61)$ & $49.32( \pm 18.81)$ & $43.17( \pm 18.38)$ & $46.58( \pm 20.84)$ \\
& CV (\%) stride time & $8.77( \pm 5.88)$ & $5.56^{* *}( \pm 3.34)$ & $8.40( \pm 5.90)$ & $4.93^{*}( \pm 3.24)$ \\
& CV (\%) step amplitude & $10.52( \pm 6.09)$ & $8.54( \pm 3.86)$ & $11.14( \pm 5.48)$ & $10.87( \pm 9.76)$ \\
Controls & $114.56( \pm 14.40)$ & $110.45^{*}( \pm 13.13)$ & $111.19^{*}( \pm 13.79)$ & $107.54^{* * *}( \pm 13.90)$ \\
& Cadence (steps/min) & $0.65( \pm 0.07)$ & $0.69^{* *}( \pm 0.08)$ & $0.67( \pm 0.10)$ & $0.68^{*}( \pm 0.09)$ \\
& Step amplitude (m) & $75.01( \pm 13.31)$ & $76.21( \pm 14.06)$ & $74.93( \pm 14.58)$ & $73.72( \pm 14.02)$ \\
& Velocity (m/min) & $2.33( \pm 0.71)$ & $2.45( \pm 0.80)$ & $3.41( \pm 1.59)$ & $2.71( \pm 1.89)$ \\
& CV (\%) stride time & $4.05( \pm 1.43)$ & $4.23( \pm 1.59)$ & $4.78( \pm 1.12)$ & $4.54( \pm 0.95)$ \\
& CV(\%) step amplitude & & & & \\
\hline
\end{tabular}

$* \mathrm{P} \leq 0.05 ; * * \mathrm{P} \leq 0.01 ; * * * \mathrm{P} \leq 0.001$ with respect to $\mathrm{PW}$ 
Cadence

ANOVA-RM indicated a significant effect of the cue $(F(3,48)=5.381 ; \mathrm{P}=0.003)$ that affected both groups in the same way (since the cue $\times$ group interaction was not significant); there were no differences between the two groups. Post hoc analysis indicated that all possible variations of the cue reduced cadence PW vs. PWA $(\mathrm{P}=0.047)$; $\mathrm{PW}$ vs. PWV $(\mathrm{P}=0.022)$; and $\mathrm{PW}$ vs. PWAV $(\mathrm{P}=0.001)$. Differences between stimulation varieties yielded significant higher cadence at PWA vs. PWAW $(\mathrm{P}=0.024)$.

Amplitude

ANOVA-RM analysis demonstrated a significant effect of the cue on the amplitude $(\mathrm{F}(1.95,31.29)=4.691 ; \mathrm{P}=0.017)$, which affected the same way both groups, (since, as with cadence, the cue $\times$ group interaction was not significant); the two groups were different $(\mathrm{F}(1,16)=18.071 ; \mathrm{P}=0.001)$. Post hoc analysis indicated that the step amplitude increased in the presence of auditory and auditoryvisual stimulation for both groups: $\mathrm{PW}$ vs. PWA $(\mathrm{P}=0.009)$; and $\mathrm{PW}$ vs. PWAV $(\mathrm{P}=0.037)$. After analysing the differences between each possible stimulation, it was found that PWA produced larger steps than PWV $(\mathrm{P}=0.01)$, while there was a tendency for PWAV to produce a larger step than PWV, although this was not significant $(\mathrm{P}=0.055)$.

\section{Velocity}

In terms of the speed at which the subjects walked, the ANOVA-RM showed a significant effect of the factor cue $(F(3,48)=3.395 ; \mathrm{P}=0.048)$ on velocity, affecting both groups in the same way (again, no significant cue $\times$ group interaction), and displaying difference between the groups $(\mathrm{F}(1,16)=14.694$; $\mathrm{P}=0.001$ ). Post hoc analysis did not yield any differences between PW and any type of stimulation. It is also noteworthy that the auditory stimulation induced faster walking than visual and auditory-visual stimulation; PWA vs. PWV (P = 0.022), PWA vs. PWAV $(\mathrm{P}=0.027)$.

CV of stride time and of step amplitude

In terms of the $\mathrm{CV}_{\text {stride-time, }}$, the ANOVA-RM showed the cue had a significant effect $(\mathrm{F}(3,14)=7.383$; $\mathrm{P}=0.003)$, which affected both groups differently as reflected by a significant cue $\times$ group interaction $(F(3,14)=5.022 ; \mathrm{P}=0.014)$. Both groups were different $(\mathrm{F}(1,16)=11.861 ; \mathrm{P}=0.003)$ and a pairwise comparison showed that there was a reduction in the $\mathrm{CV}_{\text {stride-time }}$ in the $\mathrm{PD}_{\text {III-IV }}$ patients in the presence of auditory $(\mathrm{P}=0.005)$ and auditory-visual $(\mathrm{P}=0.014)$ stimuli with respect to $\mathrm{PW}$. In contrast, no differences were seen in the control subjects. Furthermore, when the differences between the types of stimulation on the patients were analysed, there were differences between PWA vs. PWV $(P=0.034)$, and PWAV vs. PWV ( $\mathrm{P}=0.006)$, as the $\mathrm{CV}_{\text {stride-time }}$ was higher in PWV in both cases.

In contrast, for $\mathrm{CV}_{\text {step-amplitude }}$ no significant effect was seen for the cue factor and a difference was observed between the groups $(\mathrm{F}(1,16)=8.730 ; \mathrm{P}=0.009)$.

\section{Stimulation at different frequencies}

Unstimulated performance: normalization considerations

There was no difference in the FW-cadence between controls $(136.71 \pm 13.75)$ and PD $_{\text {III-IV patients }}$ $(123.93 \pm 12.84)(\mathrm{t}(16)=2.038 ; \mathrm{P}>0.05)$, this measure being used as the reference $(100 \%)$ to set the stimulation frequencies; neither in the percentage of FW-cadence corresponding to PW-cadence for each group $\left(\mathrm{t}(16)=1.196 ; \mathrm{P}>0.05 ; 89.12 \% \pm 7.66\right.$ for $\mathrm{PD}_{\mathrm{III}-\mathrm{IV}}$, and $84.15 \% \pm 9.81$ for controls $)$. 


\section{Stimulation conditions}

Cadence

ANOVA-RM analysis for auditory stimulation showed a main effect of the factor frequency on the cadence $(\mathrm{F}(2.37,37.98)=84.556 ; \quad \mathrm{P} \leq 0.001)$, affecting both groups similarly (there was no frequency $\times$ group interaction $(\mathrm{F}(2.37,37.98)=1.125 ; \mathrm{P}=0.343)$; and no difference between the groups $(F(1,16)=3.94 ; P=0.064)$. Pairwise comparisons showed that there were differences between the PW and most of the stimulation frequencies ( $\mathrm{P} \leq 0.001)$, (except $90 \% ; \mathrm{P}=0.910$, Table 6$)$.

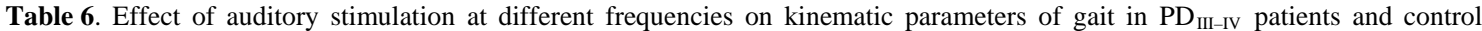
subjects

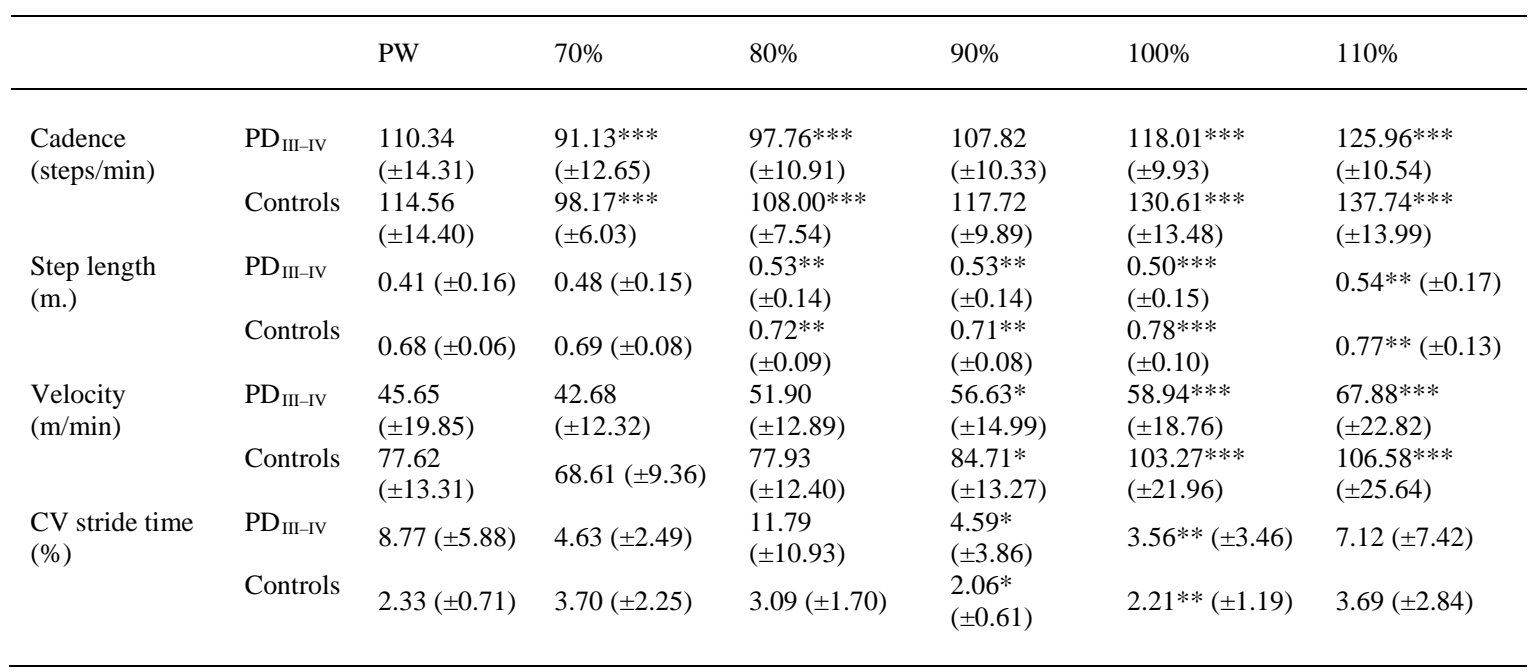

$* \mathrm{P} \leq 0.05 ; * * \mathrm{P} \leq 0.01 ; * * * \mathrm{P} \leq 0.001$ with respect to $\mathrm{PW}$

In contrast, the frequency of visual stimulation $(\mathrm{F}(2.81,44.99)=9.097 ; \mathrm{P} \leq 0.001)$ affected groups differently, as there was a significant interaction between frequency $\times$ group $(F(2.81,44.99)=3.583$; $\mathrm{P}=0.023)$. No differences were manifest between groups $(\mathrm{F}(1,16)=0.391 ; \mathrm{P}=0.541)$. Since the groups did not differ, but there was a frequency $\times$ group interaction, each group was analysed separately. There was no effect of the frequency of this stimulation on cadence in $\mathrm{PD}_{\text {III-IV }}$ patients $(\mathrm{F}(5,40)=2.835$; $\mathrm{P}=0.167)$. Conversely, in the control subjects the frequency affected cadence $(\mathrm{F}(2.68,21.48)=11.326$; $\mathrm{P} \leq 0.001)$ and a post hoc analysis indicated significant differences between $\mathrm{PW}$ and $110 \%(\mathrm{P}=0.027)$, PW and 100\% ( $\mathrm{P}=0.025)$, and $\mathrm{PW}$ and $70 \%(\mathrm{P}=0.022$; Table 7$)$.

Amplitude

The ANOVA-RM indicated that the frequency of auditory stimulation affected step amplitude $(\mathrm{F}(2.88,46.22)=9.122 ; \mathrm{P} \leq 0.001)$, which affected both groups similarly, again there being no frequency $\times$ group interaction $(F(2.88,46.22)=2.227 ; \mathrm{P}=0.100)$. Differences between the groups were evident $(\mathrm{F}(1,16)=16.972 ; \mathrm{P}=0.001)$ and the post hoc analysis indicated an increase of amplitude at the frequencies of $80 \%(P=0.003), 90 \%(P=0.002), 100 \%(P \leq 0.001)$, and $110 \%(P=0.002)$ (Table 6).

An effect of the frequency of the visual stimulus on step amplitude was also detected $(F(5,80)=3.543 ; \quad P=0.034)$, and there was no frequency $\times$ group interaction $(F(5,80)=0.356$; $\mathrm{P}=0.868)$; the two groups differed $(\mathrm{F}(1,16)=18.939 ; \mathrm{P} \leq 0.001)$. Post hoc analysis indicated that the subjects increased their amplitude at all frequencies tested except $70 \%(110 \%, \mathrm{P}=0.001 ; 100 \%$, $\mathrm{P}=0.003 ; 90 \%, \mathrm{P}=0.004$, and $80 \%, \mathrm{P}=0.004$; Table 7). 
Velocity

ANOVA-RM showed that the auditory frequency affected velocity $(\mathrm{F}(2.30,36.80)=29.420$; $\mathrm{P} \leq 0.001$ ) and this effect was similar for both patients and Controls in both groups since there was no frequency $\times$ group interaction $(\mathrm{F}(2.30,36.80)=2.883 ; \mathrm{P}=0.062)$. These groups differed in their responses $(\mathrm{F}(1,16)=21.455 ; \mathrm{P} \leq 0.001)$ and the post hoc analysis indicated differences between PW and frequencies: $110 \%(\mathrm{P} \leq 0.001), 100 \%(\mathrm{P} \leq 0.001)$, and $90 \%(\mathrm{P}=0.014)$ (Table 6$)$.

An effect of the frequency of the visual stimulation on velocity $(F(3.25,52.07)=8.254 ; \mathrm{P} \leq 0.001)$ was observed, but not a frequency $\times$ group interaction $(\mathrm{F}(3.25,52.07)=1.597 ; \mathrm{P}=0.198)$. There were different responses in each group $(\mathrm{F}(1,16)=19.885 ; \mathrm{P} \leq 0.001)$ and post hoc analysis showed differences between $\mathrm{PW}$ and the frequencies of $110 \%(\mathrm{P} \leq 0.001), 100 \%(\mathrm{P}=0.002), 90 \%(\mathrm{P}=0.01)$, and $80 \%$ $(\mathrm{P}=0.025)$ (Table 7).

Table 7. Effect of visual stimulation at different frequencies on kinematic parameters of gait in PD ${ }_{\text {III-IV }}$ patients and controls subjects

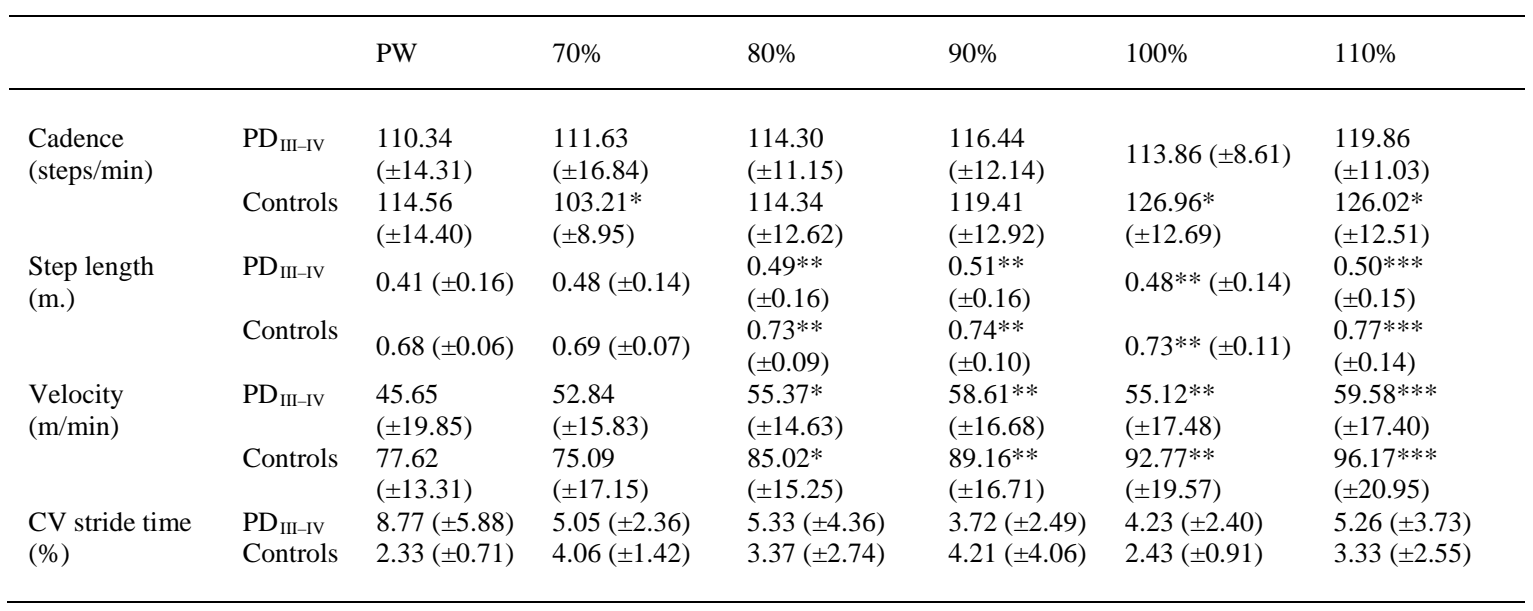

* $\mathrm{P} \leq 0.05$; ** $\mathrm{P} \leq 0.01 ; * * * \mathrm{P} \leq 0.001$ with respect to $\mathrm{PW}$

CV of stride time

The different frequencies of the auditory stimulus affected the $\mathrm{CV}_{\text {stride-time }}(\mathrm{F}(5,80)=3.546$; $\mathrm{P}=0.034$ ), in the same way in both groups (no frequency $\times$ group interaction, $(\mathrm{F}(5,80)=2.817$; $\mathrm{P}=0.066)$ ). The groups were different $(\mathrm{F}(1,16)=11.314 ; \mathrm{P}=0.004)$; and post hoc analysis showed a reduction in $\mathrm{CV}_{\text {stride-time }}$ with respect to the $\mathrm{PW}$ for frequencies of $100 \%(\mathrm{P}=0.008)$, and $90 \%(\mathrm{P}=0.042$, Table 6).

The frequency of visual stimulation did not yield any effect on the $\mathrm{CV}_{\text {stride-time }}(\mathrm{F}(5,80)=1.614$; $\mathrm{P}=0.230)$, and the groups were different $(\mathrm{F}(1,16)=4.533 ; \mathrm{P}=0.049$, Table 7$)$.

Modulation of cadence in presence of stimulation

When modulation of cadence in presence of the stimuli was analysed, the ANOVA showed a main effect of factor frequency $(\mathrm{F}(1.82,29.17)=76.827 ; \mathrm{P} \leq 0.001)$, as well as significant interaction frequency $\times$ group $(\mathrm{F}(1.82,29,17)=4.322 ; \mathrm{P}=0.026)$. A main effect for factor cue was also seen $(\mathrm{F}(1,16)=6.699 ; \mathrm{P}=0.020)$, as well as significant interactions cue $\times$ group $(\mathrm{F}=(1,16)=8.681$; $\mathrm{P}=0.009)$. Groups did not differ $(\mathrm{F}=(1,16)=2.088 ; \mathrm{P}=0.168)$.

For the $\mathrm{PD}_{\text {III-IV }}$ a main effect for factor frequency was seen $(\mathrm{F}(1.70,13.67)=23.197 ; \mathrm{P} \leq 0.001)$, as well as for factor cue $(F(1,8)=15.093 ; P=0.005)$, and interaction cue $\times$ frequency $(F(4,32)=20.555$; $\mathrm{P} \leq 0.001$ ), indicating a different performance in presence of both auditory and visual stimulation (Fig. 1). Conversely, for controls, a main effect for factor frequency $(\mathrm{F}(1.84,14.76)=59.935 ; \mathrm{P} \leq 0.001)$ was seen, but not for factor cue $(\mathrm{F}(1,8)=0.065 ; \mathrm{P}=0.805)$, indicating a lack of difference regarding stimulation variety (Fig. 1). 

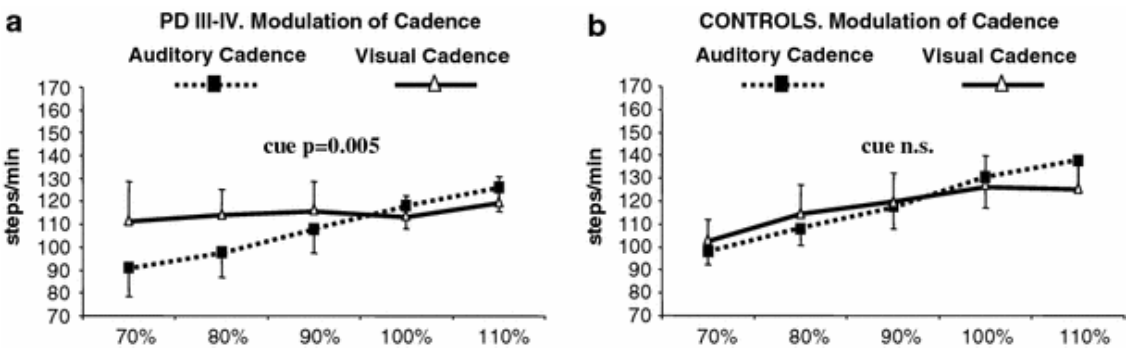

Fig. 1. Cadence induced by auditory (dotted line) and visual (solid line) stimulation in the presence of different stimulation frequencies in PD $_{\text {III-IV }}$ patients (a), and in controls (b)

\section{Discussion}

\section{Characterization of gait patterns}

As expected, our results showed that PD patients display an abnormal walking pattern involving shorter steps and slower velocity. The pattern of walking in PD patients is more variable than that of the

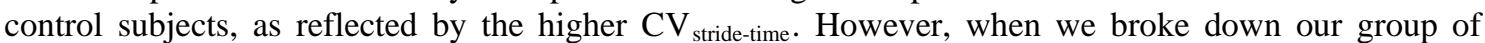
patients in order to analyse them as a function of disease development, some interesting findings appeared. Firstly, no differences were detected in any of the variables between $\mathrm{PD}_{\mathrm{I}-\mathrm{II}}$ and control subjects. This indicates that the differences in the step length, velocity, and $\mathrm{CV}_{\text {stride-time }}$ between PD patients and control subjects can be attributed to the alterations in the $\mathrm{PD}_{\text {III-IV }}$ group. Indeed, these variables were significantly altered in the PD patients when the analysis was carried out between PD III-IV $_{\text {IV }}$ and controls. In addition, the $\mathrm{CV}_{\text {step-amplitude }}$ also differed between the $\mathrm{PD}_{\mathrm{III}-\mathrm{IV}}$ and control subjects. It is also noteworthy that the cadence remained the same in patients and control subjects, regardless of whether all the patients were analysed together, or whether they were considered in separate groups. This confirms previous studies in which cadence has been shown not to be altered in PD (Morris et al. 1994a, 1998).

On the other hand, our results confirm that there are significant alterations in walking as the disease progresses, demonstrating differences between $\mathrm{PD}_{\mathrm{I}-\mathrm{II}}$ and $\mathrm{PD}_{\mathrm{III}-\mathrm{IV}}$ in terms of step amplitude, velocity,

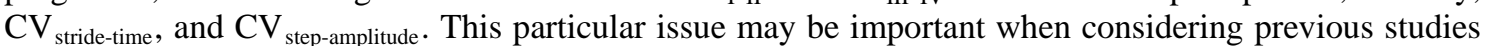
in which no distinction was made in the disease state within the "patient" sample. This is also important when contemplating studies that have assessed how sensory stimulation might aid gait in PD.

In our study, step amplitude and velocity were reduced in PD patients when compared to control subjects, in agreement with previous publications (Blin et al. 1990; Morris et al. 1994a, 1996, 2005; Thaut et al. 1996; Azulay et al. 1999; Ebersbach et al. 1999; Lewis et al. 2000; Fernández del Olmo and Cudeiro 2005; Rochester et al. 2005; Willems et al. 2006). This difference was attributed to the PD ${ }_{\text {III-IV }}$ patients, since no differences were manifested between $\mathrm{PD}_{\mathrm{I}-\mathrm{II}}$ and control subjects, in contrast to a reduction in step length in early PD as described previously (Ebersbach et al. 1999). The reduction in velocity in PD patients as a whole and in the PD $\mathrm{PII}_{\text {II IV }}$ group was the product of the shorter step amplitude, as cadence was no different between any of the patients and control subjects, in accordance with previous data (Morris et al. 1994a, 1996, 1998).

Our results regarding the $\mathrm{CV}_{\text {stride-time }}$ confirm previous studies showing that this parameter augments in PD patients (Hausdorff et al. 1998; Fernández del Olmo and Cudeiro 2005). The failure to identify differences in this variable between patients and control subjects in an earlier study may reflect the influence of early PD in that sample (Ebersbach et al. 1999). Furthermore, a recent association was reported between $\mathrm{CV}_{\text {stride-time }}$ and falls (Schaafsma et al. 2003), and falling normally occurs in the more advanced stages of PD where the highest $\mathrm{CV}_{\text {stride-time }}$ values were recorded in our study. Analysing the PD group as a function of disease development also identified an alteration in the $\mathrm{CV}_{\text {step-amplitude }}$ in the $\mathrm{PD}_{\text {III-IV }}$ patients, which was not detected when the PD patients were considered as a single group. Indeed, the fact

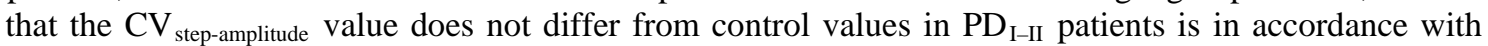
previous work (Ebersbach et al. 1999).

\section{Effect of sensory stimulation on gait}

The results obtained when the effects of sensory stimuli were analysed indicate that sensory stimulation affects similarly to $\mathrm{PD}_{\mathrm{III}-\mathrm{IV}}$ and controls, with the exception of $\mathrm{CV}_{\text {stride-time }}$ which was reduced 
only for $\mathrm{PD}_{\mathrm{III-IV}}$ if auditory or visual stimulation were employed. This means that auditory stimulation at a frequency matching the PW-cadence is effective in facilitating walking in $\mathrm{PD}_{\text {III-IV }}$ patients. The increase observed in step amplitude is important because amplitude is the variable characteristically impaired in $\mathrm{PD}$. Furthermore, the decrease in $\mathrm{CV}_{\text {stride-time }}$ might reduce the risk of falling, as $\mathrm{CV}_{\text {stride-time }}$ and the number of falls are associated (Schaafsma et al. 2003). It is also noticeable that sensory stimulation of any kind reduced the step cadence, both in $\mathrm{PD}_{\mathrm{III}-\mathrm{IV}}$ and in control subjects.

Our results concerning step amplitude are in line with previous studies that indicate a positive effect of auditory stimulation in PD, producing an increase in amplitude (Enzensberger and Fischer 1996; McIntosh et al. 1997). However, these studies often employ stimulation frequencies faster than baseline, so that the effect on amplitude may not only be attributed solely to the stimulation itself, but also to its frequency. Fewer studies have looked at the influence of stimulation that matches the PW-cadence and among these increases in step amplitude are not always observed (Rochester et al. 2005; Willems et al. 2006), although in the study carried out by Rochester et al. (2005) no explanation was offered as to why the velocity in the PD group given auditory stimulation was not equal to the reported cadence multiplied by the amplitude, perhaps suggesting an underestimation of the amplitude.

In accordance with earlier studies, visual stimulation of the type employed here did not change step amplitude (Rochester et al. 2005), although there have been some reports of an increase in stride amplitude in presence of visual stimuli (Morris et al. 1994a, 1996, 2005; Azulay et al. 1999; Lewis et al. 2000; Suteerawattananon et al. 2004). Notably, spatial visual stimuli were used in these latter studies (e.g. stripes laid out on the floor) whereas our visual stimuli were temporal visual stimuli (flashing LED's). This methodological difference probably accounts for the differences in the results observed.

Cadence was reduced by the presence of sensory stimulation in both groups and this reduction was observed across the three types of sensory stimuli tested. Conversely, auditory stimulation at this frequency increases cadence in PD patients (Freedland et al. 2002; Willems et al. 2006) and in control subjects (Willems et al. 2006).

Stimulation did not produce a change in velocity, either in $\mathrm{PD}_{\mathrm{III-IV}}$ patients or in control subjects, velocity reflecting the interaction between cadence and amplitude. The effect of the cues on cadence is compensated for by the effect of the cues on amplitude. This failure to observe a change in velocity is in agreement with previous studies using auditory stimulation (Freedland et al. 2002; Rochester et al. 2005; Willems et al. 2006). When simultaneous auditory-visual stimulation was provided, the effect on step amplitude and $\mathrm{CV}_{\text {stride-ime }}$ was similar to that of auditory stimulation alone. There did not seem to be any augmentation of the effectiveness of the auditory alone stimulation and it remains the best choice at this point, as reported previously from analysing movements in healthy subjects in paradigms other than walking (Chen et al. 2002). Indeed, in terms of velocity, the combination of both stimulation varieties led to poorer results than auditory alone for both groups.

\section{Optimization of stimulation frequencies}

Again, the effect of stimulation affected similarly to $\mathrm{PD}_{\text {III-IV }}$ and controls in many variables, with the exception of cadence in presence of visual stimulation. Willems et al. (2006) have shown similar results in presence of auditory stimulation in some variables. Howe et al. (2003) also used a gradient of auditory stimuli but their study was lacking of control group.

The analysis of the uses of different stimulation frequencies indicates that the frequency to be used in order to facilitate gait in $\mathrm{PD}_{\text {III-IV }}$ should be equal to the PW-cadence or higher, up to that matching FWcadence, but not below the PW-cadence frequency.

For $\mathrm{PD}_{\text {III-IV }}$ patients, the PW-cadence was equivalent to $89 \%$ of the FW-cadence. Accordingly, when auditory stimulation was set at $90 \%$ of the FW-cadence $\mathrm{PD}_{\text {III-IV }}$ patients increased their step length and reduced $\mathrm{CV}_{\text {stride-time, }}$ similar to the results with stimulation that matched the $\mathrm{PW}$-cadence.

Moreover, 90 and 100\% auditory stimulation increased the velocity in $\mathrm{PD}_{\text {III-IV }}$ patients and controls with respect to the PW, which becomes very important due to the slow velocity of PD patient at these stages. The $110 \%$ auditory stimulation increased step length and velocity in $\mathrm{PD}_{\text {III-IV }}$ patients and controls, but did not alter $\mathrm{CV}_{\text {stride-time. }}$. Similarly, $80 \%$ auditory stimulation also induced larger steps in both groups, although this frequency was the only one that might increase $\mathrm{CV}_{\text {stride-time }}$ (although not significantly) in $\mathrm{PD}_{\text {III-IV }}$. As $\mathrm{CV}_{\text {stride-time }}$ has been positively associated with falls in PD (Schaafsma et al. 2003) we do not recommend the use of this frequency, in contrast to other studies (Willems et al. 2006).

Application of a range of auditory stimuli to a group of PD patients has also been proposed previously (Willems et al. 2006). However, surprisingly no comparison was made with PW and thus, no conclusions can be drawn regarding the facilitatory effect that auditory stimulation might have on the impaired PW in PD patients. 
Cadence was modulated differently depending on the kind of stimulation. Auditory stimulation led to an increase in cadence with respect to the PW at higher frequencies (100 and 110\%). However, when the frequency was slow (80 and 70\%) the cadence was lower than the PW in both groups. Since the $90 \%$ frequency was close to the PW-cadence it did not change the cadence with regards PW. Conversely, no frequency of visual stimulation altered the cadence with regard to the $\mathrm{PW}$ for the $\mathrm{PD}_{\text {III-IV }}$ patients. This behaviour was not so marked in the control subjects, whose cadence increased with faster stimulation frequencies (100 and 110\%) and diminished at $70 \%$. As indicated previously for movements other than walking (Chen et al. 2002), this suggests a more powerful interaction between motor and auditory systems than motor and visual systems. This different interaction seems to be enhanced in PD patients, and it is confirmed by the fact that the cue factor is significant in $\mathrm{PD}_{\text {III-IV }}$ patients when comparing the performance of cadence with the auditory and visual frequencies proposed. This must be taken into account when interpreting other variables dependent on cadence such as velocity. At low frequency stimulation, the velocity might be faster with visual cues rather than auditory stimulation. However, this may not reflect a better facilitation of the visual stimuli but rather a poorer adaptation to the frequency proposed. Unfortunately, there is lack of studies using different frequencies of visual stimulation in PD patients. However, this is not the case for auditory stimuli where our data regarding the modulation of cadence in the presence of different auditory frequencies above and below baseline are in line with those of Howe et al. (2003). Auditory frequencies above the PW-cadence (5\%) did not modify cadence in $\mathrm{PD}_{\text {III- }}$ Iv patients in another study (Enzensberger and Fischer 1996), probably due to the proximity between the stimulus frequency and the PW-cadence. This result again fits well with our data, as $90 \%$ auditory stimulation did not produce a change in cadence in $\mathrm{PD}_{\text {III-IV }}$ patients. Other authors have proposed auditory stimulation at different frequencies (McIntosh et al. 1997; Suteerawattananon et al. 2004), although the interpretation of the results becomes more difficult as the frequencies proposed were compared with FW.

Auditory stimulation, at a frequency of 80, 90, 100 and $110 \%$, induces larger steps in PD $_{\text {III-IV }}$ patients and controls with regard to PW. This is important as short-step amplitude is the typical feature of PD (Morris et al. 1994a, 1998). The increase in step length at $90 \%$ is in line with our results when the stimulation provided matched the $\mathrm{PW}$-cadence, where $\mathrm{PD}_{\text {III-IV }}$ patients increased their amplitude in the presence of auditory stimulation at this frequency (89\%). The increment of amplitude at frequencies equivalent to $80 \%$ in PD has also been found by others (Willems et al. 2006) and an increase in step amplitude in $\mathrm{PD}_{\text {III-IV }}$ patients was also observed when synchronizing auditory stimuli at a frequency slightly above PW-cadence (Enzensberger and Fischer 1996). However, stride length does not appear to be modified in the same way in mildly affected PD patients (Howe et al. 2003). Other studies (McIntosh et al. 1997; Suteerawattananon et al. 2004) reported contradictory results in step amplitude in the presence of auditory stimulation at different frequencies, which could be explained by the amplitude-cadence relationship (Morris et al. 1998). However, in these studies the baseline condition was FW, which in our opinion is not relevant to the results with respect to the everyday-PW.

Auditory stimulation at a frequency of 90 and $100 \%$ reduced the $\mathrm{CV}_{\text {stride-time }}$ in $\mathrm{PD}_{\text {III-IV }}$ patients and controls. The reduction at $90 \%$ is in agreement with our results for $\mathrm{PD}_{\mathrm{III}-\mathrm{IV}}$ in experiments using auditory cues at a frequency matching the PW-cadence (89\%). Auditory stimulation at $80 \%$ (frequency below PWcadence) slightly increased $\mathrm{CV}_{\text {stride-time }}$ in $\mathrm{PD}_{\text {III-IV }}$ patients, results that are in line with the increase in this variable with stimulation at a frequency 20\% slower than PW-cadence (Ebersbach et al. 1999) and other similar results (Willems et al. 2006).

Visual stimulation had no effect on $\mathrm{CV}_{\text {stride-time, }}$, neither in $\mathrm{PD}_{\text {III-IV }}$ patients nor in control subjects.

Why auditory stimulation modified gait parameters in $\mathrm{PD}_{\text {III-IV }}$ patients and controls, especially at frequencies matching the PD cadence, is beyond the scope of this study. Previous work from our group indicated a higher cerebellar metabolism (using PET) at rest in PD patients following therapy based on auditory stimulation at different frequencies (Fernández del Olmo et al. 2006). Moreover, we identified changes in kinematic parameters of walking. This is line with Goldberg's theory, which associates SMA with self-paced movements, and PMA to externally triggered movements (Goldberg 1985). According to this theory, the use of sensory cues in the execution of movement by PD patients would be translated into less involvement of the defective areas. However, this theory would not explain the different behaviour in the presence of auditory or visual stimulation. An effect of auditory stimulation on the excitability of motoneurons has been demonstrated (Rossignol and Melvill-Jones 1976) and this could account for such differences, and might also explain the control's behaviour. However, more research into the interaction between visual and auditory stimulation in the higher structures ruling movement is needed to clarify the behaviour shown by PD patients in the presence of visual or auditory stimulation. 


\section{Conclusions}

In summary, this study indicates that while our $\mathrm{PD}_{\text {I-II }}$ group do not display a significant impairment in the kinematic variables, we have studied the $\mathrm{PD}_{\text {III-IV }}$ patients presented abnormal step amplitude,

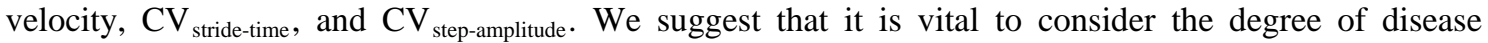

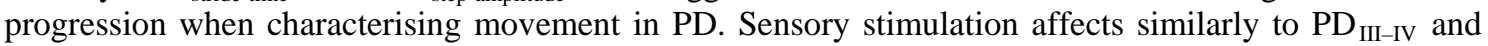
controls in a number of variables, suggesting that an effect on early PD might also be present. Auditory stimulation at a frequency matching PW-cadence is an effective tool, which facilitates improvement in gait in advanced PD patients. This kind of stimulation increases step amplitude and reduces $\mathrm{CV}_{\text {stride-time, }}$ which is most important given its relationship to falling. However, visual stimulation at this frequency does not appear to have the same influence on the kinematic parameters analysed in this study, but the restricted nature of the visual stimulation should be noted (temporally not spatially modulated; fixed to head, not retinal location). Together with experiments using different stimulation frequencies we conclude that the frequency that should be used ranges from that matching the patient's cadence in the absence of stimulation, up to a frequency matching FW-cadence. This range of auditory frequencies

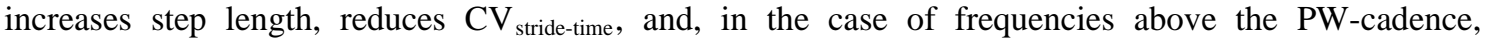
augments velocity. In contrast to other studies (Willems et al. 2006), auditory frequencies slower than the

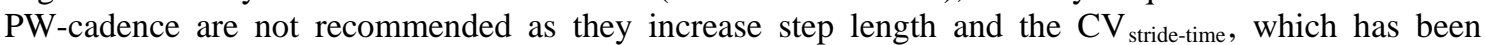
associated with falls (Schaafsma et al. 2003).

Acknowledgments. We would like to thank K.L. Grieve for his comments and corrections on the manuscript, M. Fernández del Olmo for his contribution to the work described here, and Salvador Naya and Ricardo Cao for their advice on statistical analysis. This study was supported by Xunta de Galicia (PGIDIT06PXIC137004PN and Consellería de Educación-2007/000140-0), Spain.

\section{References}

Azulay JP, Mesure S, Amblard B, Blin O, Sangla I, Pouget J (1999) Visual control of locomotion in Parkinson's disease. Brain 122:111-120

Blin O, Ferrandez AM, Serratrice G (1990) Quantitative analysis of gait in Parkinson patients: increase variability of stride length. J Neurol Sci 98(1):91-97

Chen Y, Repp BH, Patel A (2002) Spectral decomposition of variability in synchronization and continuation tapping: comparison between auditory and visual pacing feedback conditions. Hum Mov Sci 21:515-532

Ebersbach G, Heijmenberg M, Kindermann L, Trottenberg T, Wissel J, Poewe W (1999) Interference of rhythmic constraint on gait in healthy subjects and patients with early Parkinson's disease: evidence for impaired locomotor pattern generation in early Parkinson's disease. Mov Disord 14(4):619-625

Enzensberger W, Fischer PA (1996) Metronome in Parkinson’s disease. Lancet 347:133

Fahn S, Elton RL (1987) Unified Parkinson's disease rating scale. In: Fahn S, Marsden CD, Calne D, Goldstein M (eds) Recent developments in Parkinson's disease. Macmillan, Florham Park, pp 153-163

Fernández del Olmo M, Cudeiro J (2005) Temporal variability of gait in PD. Effects of a rehabilitation programme based on rhythmic sound cues. Parkinsonism Relat Disord 11:25-33

Fernández del Olmo M, Arias P, Furio MC, Pozo MA, Cudeiro J (2006) Evaluation of the effect of training using auditory stimulation on rhythmic movement in Parkinsonian patients-a combined motor and [18F]-FDG PET study. Parkinsonism Relat Disord 12:154-164

Freedland RL, Festa C, Sealy M, McBean A, Elghazaly P, Capan A, Brozycki L, Nelson AJ, Rothman J (2002) The effects of pulsed auditory stimulation on various gait measurements in persons with Parkinson's disease. NeuroRehabilitation 17:81-87

Frenkel-Toledo S, Giladi N, Peretz C, Herman T, Gruendlinger L, Hausdorff JM (2005) Effect of gait speed on gait rhythmicity in Parkinson's disease: variability of stride time and swing time respond differently. J NeuroEng Rehabil 2:23-29

Goetz CG, Poewe W, Rascol O, Sampaio C, Stebbins GT, Counsell C, Giladi N, Holloway RG, Moore CG, Wenning GK, Yahr MD, Seidl L (2004) Movement Disorder Society Task Force report on the Hoehn and Yahr staging scale: status and recommendations. Mov Disord 19(9):1020-1028

Goldberg G (1985) Supplementary motor area structure and function: review and hypotheses. Behav Brain Res 8:567-588

Hausdorff JM, Cudkowicz ME, Firtion R, Wei JY, Goldberger AL (1998) Gait variability and basal ganglia disorders: stride-to-stride variations of gait cycle timing in Parkinson's disease and Huntington's disease. Mov Disord 13(3):428-437

Hoehn MM, Yahr MD (1967) Parkinsonism: onset, progression, and mortality. Neurology 17:427-42

Howe TE, Lovgreen B, Cody FW, Ashton VJ, Oldham JA (2003) Auditory cues can modify the gait of persons with early-stage Parkinon's disease: a method for enhancing parkinsonian walking performance? Clin Rehabil 17(4):363-367 
Kokmen E, Naessens JM, Offord KP (1987) A short test of mental status: description and preliminary results. Mayo Clin Proc 62:281-288

Lewis GN, Byblow WD, Walt SE (2000) Stride length regulation in Parkinson's disease: the use of extrinsic, visual cues. Brain 123:2077-2090

Marchese R, Diverio M, Zucchi F, Lentino C, Abbruzzese G (2000) The role of sensory cues in the rehabilitation of parkinsonian patients: a comparison of two physical therapy protocols. Mov Disord 15(5):879-883

McIntosh GC, Brown SH, Rice RR, Thaut MH (1997) Rhythmic auditorymotor facilitation of gait patterns in patients with Parkinson’s disease. J Neurol Neurosurg Psychiatry 62:22-26

Miller RA, Thaut MH, McIntosh GC, Rice RR (1996) Components of EMG symmetry and variability in parkinsonian and healthy elderly gait. Electroencephalogr Clin Neurophysiol 101:1-7

Morris M, Iansek R, Matyas TA, Summers JJ (1998) Abnormalities in the stride length-cadence relation in Parkinsonian gait. Mov Disord 13:61-69

Morris M, Iansek R, McGinley J, Matyas T, Huxham F (2005) Three-dimensional gait biomechanics in Parkinson’s disease: evidence for centrally mediated amplitude regulation disorder. Mov Disord 20(1):40-50

Morris ME, Iansek R, Matyas TA, Summers JJ (1994a) Ability to modulate walking cadence remains intact in Parkinson's disease. J Neurol Neurosurg Psychiatry 57:1532-1534

Morris ME, Iansek R, Matyas TA, Summers JJ (1994b) The pathogenesis of gait hypokinesia in Parkinson's disease. Brain 117:1169-1181

Morris ME, Iansek R, Matyas TA, Summers JJ (1996) Stride length regulation in Parkinson's disease. Normalization strategies and underlying mechanisms. Brain 119:551-568

Nieuwboer A, Kwakkel G, Rochester L, Jones D, van Wegen E, Willems AM, Chavret F, Hetherington V, Baker K, Lim I (2007) Cueing training in the home improves gait-related mobility in Parkinson's disease: the RESCUE trial. J Neurol Neurosurg Psychiatry 78:134-140

Rochester L, Hetherington V, Jones D, Nieuwboer A, Willems AM, Kwakkel G, Van Wegen E (2005) The effect of external rhythmic cues (auditory and visual) on walking during a functional task in homes of people with Parkinson's disease. Arch Phys Med Rehabil 86:999-1006

Rossignol S, Melvill-Jones G (1976) Audiospinal influences in man studied by the H-reflex and its possible role in rhythmic movement synchronized to sound. Electroencephalogr Clin Neurophysiol 41:83-92

Schaafsma JD, Giladi N, Balash Y, Bartels AL, Gurevich T, Hausdorff JM (2003) Gait dynamics in Parkinson's disease: relationship to Parkinsonian features, falls and response to levodopa. J Neurol Sci 212:47-53

Schwab RS, England AC (1969) Projection technique for evaluating surgery in Parkinson's disease. In: Gillingham FJ, Donaldson IML (eds) Third symposium on Parkinson's disease. Livingstone, Edinburgh, pp 152-157

Suteerawattananon M, Morris GS, Etnyre BR, Jankovic J, Protas EJ (2004) Effect of visual and auditory cues on gait in individuals with Parkinson's disease. J Neurol Sci 219:63-69

Thaut MH, McIntosh GC, Rice RR, Miller RA, Rathbun J, Brault JM (1996) Rhythmic auditory stimulation in gait training for Parkinson's Disease patients. Mov Disord 11(2):193-200

Willems AM, Nieuwboer A, Chavret F, Desloovere K, Dom R, Rochester L, Jones D, Kwakkel G, Van Wegen E (2006) The use of rhythmic auditory cues to influence gait in patients with Parkinson's disease, the differential effect for freezers and non-freezers, an explorative study. Disabil Rehabil 28(11):721-728 